\title{
Impact of glucose on risk of dementia: Mendelian randomisation studies in 115,875 individuals
}

\author{
Marianne Benn $^{1,2,3}$ (D) Børge G. Nordestgaard ${ }^{2,3,4,5}$ (D) A Anne Tybjærg-Hansen ${ }^{1,2,3,5} \cdot$ Ruth Frikke-Schmidt $^{1,2,3,5}$ (D)
}

Received: 13 December 2019 / Accepted: 14 February 2020 / Published online: 14 March 2020

(C) Springer-Verlag GmbH Germany, part of Springer Nature 2020

\begin{abstract}
Aims/hypothesis Mendelian randomisation studies have not shown a clear causal effect of high plasma glucose on the risk of Alzheimer's disease. We tested the hypothesis that high plasma glucose caused by genetic variation has a causal effect on the risk of unspecified dementia, Alzheimer's disease and vascular dementia in the general population.

Methods A Mendelian randomisation design was used with data from 115,875 individuals from the Copenhagen General Population Study and the Copenhagen City Heart Study. Findings for Alzheimer's disease were validated in a two-sample Mendelian design with 455,258 individuals, including 71,880 individuals with Alzheimer's disease or a parent with Alzheimer's disease.

Results In observational multifactorial-adjusted analyses, HRs were 1.15 (95\% CI 1.01, 1.32; $p=0.039$ ) for unspecified dementia, $0.91(95 \%$ CI $0.79,1.06 ; p=0.22)$ for Alzheimer's disease and 1.16 (95\% CI 0.86, $1.55 ; p=0.34)$ for vascular dementia in individuals with a glucose level higher than 7 vs 5-6 mmol/l. Corresponding HRs in individuals with vs without type 2 diabetes were $1.42(95 \%$ CI 1.24, 1.63; $p<0.001), 1.11$ (95\% CI 0.95, 1.29; $p=0.18)$ and 1.73 (95\% CI $1.31,2.27 ; p<0.001)$. In genetic causal analyses, a $1 \mathrm{mmol} / 1$ higher plasma glucose level had RRs of 2.40 (95\% CI 1.18, 4.89; $p=0.016)$ for unspecified dementia, 1.41 (95\% CI 0.82, 2.43; $p=0.22$ ) for Alzheimer's disease and 1.20 (95\% CI 0.82, $1.75 ; p=0.36)$ for vascular dementia. Summary-level data from the Meta-Analyses of Glucose and Insulin-related Traits Consortium (MAGIC) combined with a consortium of the Alzheimer's Disease Sequencing Project (ADSP), the International Genomics of Alzheimer's Project (IGAP), the Alzheimer's Disease Working Group of the Psychiatric Genomics Consortium (PGC-ALZ) and the UK Biobank (UKB) gave an RR for Alzheimer's disease of 1.02 (95\% CI 0.92, 1.13; $p=0.42$ ), and this consortium combined with Copenhagen studies gave an RR for Alzheimer's disease of 1.03 (95\% CI 0.93, 1.13; $p=0.36)$.

Conclusions/interpretation Observational and genetically high plasma glucose are causally related to the risk of unspecified dementia, but not to Alzheimer's disease or vascular dementia.
\end{abstract}

Keywords Alzheimer's disease · Diabetes mellitus · Glucose $\cdot$ Mendelian randomisation · Unspecified dementia · Vascular dementia

Electronic supplementary material The online version of this article (https://doi.org/10.1007/s00125-020-05124-5) contains peer-reviewed but unedited supplementary material, which is available to authorised users.

Marianne Benn

Marianne.benn@regionh.dk

1 Department of Clinical Biochemistry, Rigshospitalet, Copenhagen University Hospital, Blegdamsvej 3, 2100 Copenhagen, Denmark

2 The Copenhagen General Population Study, Herlev and Gentofte Hospital, Copenhagen University Hospital, Copenhagen, Denmark
3 Faculty of Health and Medical Sciences, Department of Clinical Medicine, University of Copenhagen, Copenhagen, Denmark

4 Department of Clinical Biochemistry, Herlev and Gentofte Hospital, Copenhagen University Hospital, Copenhagen, Denmark

5 The Copenhagen City Heart Study, Frederiksberg Hospital, Copenhagen University Hospital, Copenhagen, Denmark 


\section{Research in context}

What is already known about this subject?

- Observational studies have reported an increased risk of dementia in individuals with diabetes

- Previous results from Mendelian randomisation studies on glycaemic traits and Alzheimer's disease are not clear, and unspecified dementia and vascular dementia have not been examined

\section{What is the key question?}

- Using Mendelian randomisation with data from 115,875 individuals from the Danish general population, does genetically high plasma glucose have a causal effect on the risk of dementia?

What are the new findings?

- A $1 \mathrm{mmol} / \mathrm{l}$ higher plasma glucose level had a causal effect with a 2.4-fold higher risk of unspecified dementia, but not a higher risk of Alzheimer's disease or vascular dementia

How might this impact on clinical practice in the foreseeable future?

- The finding that high plasma glucose is causally related to the risk of unspecified dementia underscores the importance of glycaemic control in patients with diabetes mellitus and potentially also in individuals with impaired glucose tolerance

\begin{tabular}{|c|c|}
\hline \multicolumn{2}{|c|}{ Abbreviations } \\
\hline ADSP & Alzheimer's Disease Sequencing Project \\
\hline IGAP & International Genomics of Alzheimer's Project \\
\hline MAGIC & Meta-Analyses of Glucose and \\
\hline & Insulin-related Traits Consortium \\
\hline PGC-ALZ & $\begin{array}{l}\text { Alzheimer's Disease Working Group } \\
\text { of the Psychiatric Genomics Consortium }\end{array}$ \\
\hline UKB & UK Biobank \\
\hline
\end{tabular}

\section{Introduction}

Observational studies have reported an association between raised plasma glucose concentrations and the risk of dementia in individuals both with and without diabetes [1]. However, patients with high plasma glucose concentrations and potentially type 2 diabetes often have several risk factors for dementia other than high plasma glucose (e.g. hypertension, hyperlipidaemia, being overweight, vascular disease), and it is not clear whether glucose per se has a causal effect on the risk of dementia, or whether the observed risks are due to confounding or reverse causation.

To investigate whether plasma glucose has a causal effect on the risk of unspecified dementia, Alzheimer's disease and vascular dementia, we used the Mendelian randomisation design, which aims to circumvent confounding and reverse causation through the use of genetic variation in populations [2-4]. The study design is very similar to that of a randomised clinical intervention trial using randomisation by genes instead of treatment and, because of the random assortment of genetic variants at conception, variants with an effect on glucose are randomly distributed in relation to potential confounders. In addition, because genetic variants are determined at conception and remain constant throughout life, the Mendelian randomisation design is not influenced by reverse causation.

Previous Mendelian randomisation studies have examined the causal effects of type 2 diabetes, insulin sensitivity and fasting glucose on the risk of Alzheimer's disease but the results have not been clear, and the risks of unspecified dementia and vascular dementia have not been examined [5-7].

Using a Mendelian randomisation design in 115,875 individuals from two prospective general population studies, the Copenhagen General Population Study and the Copenhagen City Heart Study [8, 9], we tested the hypothesis that high plasma glucose caused by genetic variation is associated with a high risk of unspecified dementia, Alzheimer's disease and vascular dementia as an indication of causality.

We tested: (1) whether non-fasting plasma glucose and type 2 diabetes at baseline are observationally associated with dementia; (2) whether a weighted allele score of plasma glucose-increasing alleles is associated with high plasma glucose concentrations, as expected; (3) whether the weighted glucose-increasing alleles are associated with the risk of dementia, as an indication of a causal effect of high glucose on the risk of dementia; and (4) whether the causal effect of high plasma glucose is consistent with the corresponding observational associations using instrumental variable analysis (see electronic supplementary material [ESM] Fig. 1). For Alzheimer's disease, we also included a risk estimate using 
summary-level data from the Meta-Analyses of Glucose and Insulin-related Traits Consortium (MAGIC), with data on 46,186 individuals without diabetes and of European ancestry [10-13], combined with the Alzheimer's Disease Working Group of the Psychiatric Genomics Consortium (PGCALZ), the International Genomics of Alzheimer's Project (IGAP) and the Alzheimer's Disease Sequencing Project (ADSP) comprising individuals with a clinical diagnosis of Alzheimer's disease and the Alzheimer's disease by proxy (parent with Alzheimer's disease) from the UK Biobank (UKB) (ADSP/IGAP/PGC-ALZ/UKB) [14] with 455,258 individuals, including 71,880 individuals with Alzheimer's disease $(34 \%$ of participants) or with a parent with Alzheimer's disease (66\% of participants), and examining the same genetic variants as in the Copenhagen studies.

\section{Methods}

Participants We included individuals from two similar prospective studies of the Danish general population: the Copenhagen General Population Study $(N=107,143)$ and the Copenhagen City Heart Study $(N=18,732)[8,9]$. Combining the two studies yielded a total of 125,875 individuals, including 115,958 with available genotypes of whom 2596 developed unspecified dementia, 2287 Alzheimer's disease and 493 vascular dementia during up to 43 years of follow-up with a median of 10 years. All individuals were white and of Danish descent, and none was included in more than one study. No individuals were lost to follow-up.

The Copenhagen General Population Study was initiated in 2003 and enrolment is ongoing [8, 9]. Individuals are selected based on the national Danish Civil Registration System to reflect the adult white Danish population at 20-100 years of age. Data are obtained from a questionnaire reviewed by the participant and an investigator on the day of attendance, a physical examination and blood samples including DNA extraction.

The Copenhagen City Heart Study is a prospective study of the Danish general population initiated in 1976-1978, with follow-up examinations in 1981-1983, 1991-1994 and 2001-2003 [8, 9]. Participants were recruited and examined exactly as in the Copenhagen General Population Study. Baseline was considered the first examination of an individual in which plasma glucose was measured.

Ethics approval Studies were approved by the Herlev and Gentofte Hospital and by Danish ethical committees (KF100.2039/91, KF-01-144/01, H-KF-01-144/01). Written informed consent was obtained from participants.

Endpoints Diagnoses of endpoints according to ICD-8 (http:// www.wolfbane.com/icd/icd8.htm) and ICD-10 (http://apps. who.int/classifications/icd10/browse/2016/en) codes were collected from 1977 to 13 December 2018 by reviewing all hospital admissions and diagnoses in the Danish National Patient Register and all causes of death in the national Danish Register of Causes of Death [15, 16]. Endpoints were defined as follows: unspecified dementia (ICD8: 290.18; ICD10: F03), Alzheimer's disease (ICD8: 290.10; ICD10: F00, G30) and vascular dementia (ICD10: F01), as done previously $[15,16]$. In Denmark, these diseases are diagnosed by specialists in neurology, and a validation with full clinical work-up of register-based dementia diagnoses concluded that diagnostic validity in the Danish hospital registries is high [17]. In the present study, the validity of the register-based dementia diagnoses was further ensured by the presence of the well-known association with the $A P O E$ (encoding apolipoprotein E) $\varepsilon 4$ allele with Alzheimer's disease in the Copenhagen General Population Study and the Copenhagen City Heart Study [16, 18].

Potential confounders and covariates Non-fasting plasma concentrations of glucose, total cholesterol, HDL-cholesterol and triacylglycerols were measured using standard hospital assays. LDL-cholesterol was calculated using the Friedewald equation if plasma triacylglycerols were $\leq 4.0 \mathrm{mmol} / 1$, and measured by a direct enzymatic method at higher triacylglycerol concentrations (Konelab autoanalyser, Thermo Fisher Scientific, San Diego, CA, USA). BMI was measured-weight divided by measured-height squared. Hypertension was defined as systolic $\mathrm{BP} \geq 140 \mathrm{mmHg}(\geq 135 \mathrm{mmHg}$ in individuals with diabetes mellitus), diastolic BP $\geq 90 \mathrm{mmHg}$ ( $\geq 85 \mathrm{mmHg}$ for diabetics) or use of antihypertensive medication prescribed specifically for hypertension [19]. Diabetes mellitus was self-reported or classified by non-fasting plasma glucose $>11.0 \mathrm{mmol} / \mathrm{l}$, medication prescribed for diabetes, and/or hospitalisation or death due to diabetes (ICD8: 249-250; ICD10: E10-11, E13-14) [20]. The use of cholesterol-lowering medication was self-reported, with $>97 \%$ being statins. Participants also reported on smoking, coded as current smokers and non-smokers. Work and leisure time physical activity was coded as 'low' for $0-2 \mathrm{~h}$ moderate activity per week and 'intermediate and high' for more than $2 \mathrm{~h}$ moderate or vigorous activity per week during either work or leisure time. Information on alcohol consumption was summarised in units per week ( 1 unit $=\sim 12 \mathrm{~g}$ alcohol) and coded as 'above recommended' if more than 14 or 21 units per week for women and men, respectively. Education was summarised as $<8$ or $\geq 8$ years of completed education. For women, menopausal status was also recorded. Missing data on covariates varied from $0 \%$ to $1 \%$ for any individual variable. Individuals with missing covariates were excluded $(<1.3 \%$ excluded $)$ from observational analyses.

Genotyping An ABI PRISM 7900HT Sequence Detection System (Applied Biosystems, Foster City, CA, USA) and TaqMan-based assays were used to genotype for the 
glucose-associated genotypes rs560887 (G6PC2), rs4607517 $(G C K)$, rs2191349 (DGKB), rs 11708067 (ADCY5), rs7903146 (TCF7L2), and rs2383206 and rs10811661 $(C D K N 2 A / C D K N 2 B)$. These genotypes have been reported to have the strongest effect on plasma glucose in genomewide association studies, their functions are well known [12, 13] and pleiotropic effects have not been observed. A weighted allele score was constructed by summing the number of glucose-increasing alleles, weighted by frequency and effect size on plasma glucose, and divided into the following categories: 0-24th percentile, 25-49th percentile, 50-74th percentile, 75-94th percentile and 95-100th percentile by the weighted allele score. Individuals included in genetic analyses had complete data on age, sex, genotypes and endpoints.

Statistical analyses Data were analysed using StataIC 14.2 [21]. For genotypes, deviation from Hardy-Weinberg equilibrium was tested using a Pearson $\chi^{2}$ test. To test whether high plasma glucose was observationally associated with the risk of disease (ESM Fig. 1, arrow 1), we used restricted cubic splines with 5 knots, selected using Akaike's information criterion to balance best fit and overfitting [22], and a glucose concentration corresponding to the population median of $5.2 \mathrm{mmol} / \mathrm{l}$ as reference. To test whether type 2 diabetes mellitus was associated with disease, we used Cox regression models with age as the time scale and adjusted for sex and year of birth; and multifactorial models further adjusted for the potential confounders BMI, hypertension, smoking, physical activity, alcohol consumption, education and (for women) menopausal status. Adjustment for year of birth was performed to accommodate changes in diagnostic criteria and treatment over calendar time. To reduce the risk of bias due to confounding (factors with an effect on both exposure and outcome) and covariates (factors with an effect on the endpoint), multifactorial adjustment was included. Trends for significance across ordered categories of high plasma glucose concentrations and allele score (see below) were tested using the non-parametric Cuzick's extension of a Wilcoxon rank-sum test.

Linear regression was used to test whether the weighted allele score was associated with a high plasma glucose concentration (ESM Fig. 1, arrow 2).

To test whether the weighted glucose allele score was associated with the risk of dementia, we used Cox regression models adjusted for age, sex and year of birth (ESM Fig. 1, arrow 3). The follow-up time for Cox regression analyses began at the first inclusion into a study and ended with censoring at the date of death, the occurrence of an event, emigration ( $n=853$ ) or on 13 December 2018 (corresponding to the date of register retrieval of diagnoses), whichever came first.

As a positive control of the genetic instrument and study power, the known association with ischaemic heart disease was included and, as a positive control on study power and endpoints, results on the known risk of Alzheimer's disease for $A P O E$ ع34/ع44 vs $\varepsilon 33$ were included $[16,18]$. As sensitivity analyses, associations were also examined excluding individuals using cholesterol-lowering medication and those with diabetes mellitus at baseline, in quintile categories and using non-Alzheimer's disease as the endpoint.

Logistic regression was used to assess whether observational high plasma glucose, the weighted allele score, $A P O E \varepsilon 34 / \varepsilon 44$, Alzheimer's disease, vascular dementia and unspecified dementia were associated with the potential confounders of age, sex, BMI, hypertension, smoking, physical activity, alcohol consumption, education and (for women) menopausal status.

Because genotypes are constant throughout life, and hence insensitive to reverse causation, a potential causal effect of genetically high plasma glucose on the risk of dementia was assessed using instrumental variable analysis (ESM Fig. 1, arrow 4) using the user-written ivreg2 and ivpois commands in Stata [23, 24]. The strength of the instruments (i.e. the strength of the association of the genotypes with plasma glucose concentration) was confirmed by $F$-statistics of $F=$ 80.7 from regressions using ivreg2, where $F>10$ was considered acceptable [2].

Finally, we conducted a summary-level Mendelian randomisation analysis $[4,25]$ using the same genetic variants as in our own studies, but now with information on the effect on plasma glucose concentrations from the publicly available MAGIC (46,186 individuals without diabetes) data [10-13], and for each glucose-increasing variant estimated the risk of Alzheimer's disease using the ADSP/IGAP/PGC-ALZ/UKB population $(455,258$ individuals, including 71,880 with Alzheimer's disease or a parent with Alzheimer's disease) [14]. For information on these study populations, please refer to ESM Methods. To verify the causal effect of high plasma glucose on the risk of Alzheimer's disease from the summarylevel data, we performed regression analysis by Egger Mendelian randomisation [26] using the user-written mregger command in Stata [27], and accounting for potential weak instruments and direct pleiotropic effects of the variants, respectively $[26,27]$. Statistical power to detect a significant ( $\alpha=0.05$ and $\beta=0.80$ ) effect of genetically determined glucose on the risk of dementia was calculated using $r^{2}=$ $0.3 \%$, a sample size of 110,000 individuals, and a frequency of endpoints of $1 \%$ and expressed as a fraction from 0 to 1.0 [28].

\section{Results}

Of 125,875 individuals included in the study, baseline nonfasting plasma glucose concentrations were $<5.0 \mathrm{mmol} / \mathrm{l}$ in $35 \%, 5.0-5.9 \mathrm{mmol} / 1$ in $42 \%, 6.0-6.9 \mathrm{mmol} / 1$ in $14 \%$ and $\geq 7.0 \mathrm{mmol} / 1$ in $8.4 \%$ (Table 1 ). Individuals with a nonfasting plasma glucose level of $\geq 7.0 \mathrm{vs}<6.0 \mathrm{mmol} / 1$ were older, more often men, had higher triacylglycerol 
Table 1 Baseline characteristics of participants by plasma glucose categories

\begin{tabular}{|c|c|c|c|c|c|c|}
\hline \multirow[b]{2}{*}{ Characteristic } & \multicolumn{4}{|c|}{ Plasma glucose, mmol/1 } & \multirow[b]{2}{*}{$p_{\text {trend }}$} & \multirow[b]{2}{*}{ All } \\
\hline & $<5.0$ & $5.0-5.9$ & $6.0-6.9$ & $\geq 7.0$ & & \\
\hline Individuals, $n(\%)$ & $44,616(35)$ & $52,678(42)$ & $17,985(14)$ & $10,596(8.4)$ & & $125,875(100)$ \\
\hline Age, years & $54(45-64)$ & $58(48-67)$ & $59(49-68)$ & $60(52-69)$ & $<0.001$ & $57(47-66)$ \\
\hline Women, $n(\%)$ & $26,323(59)$ & $28,446(54)$ & $9352(52)$ & $4768(45)$ & $<0.001$ & $69,231(55)$ \\
\hline Glucose, mmol/1 & $4.6(4.4-4.8)$ & $5.3(5.1-5.6)$ & $6.3(6.1-6.6)$ & $7.8(7.3-8.8)$ & $<0.001$ & $5.2(4.8-5.9)$ \\
\hline LDL-cholesterol, mmol/1 & $3.2(2.6-3.8)$ & $3.3(2.6-3.9)$ & $3.3(2.6-4.0)$ & $3.1(2.4-3.9)$ & $<0.001$ & $3.2(2.6-3.9)$ \\
\hline Triacylglycerols, mmol/1 & $1.2(0.9-1.8)$ & $1.4(1.0-2.1)$ & $1.6(1.1-2.3)$ & $1.7(1.2-2.6)$ & $<0.001$ & $1.4(1.0-2.1)$ \\
\hline HDL-cholesterol, mmol/l & $1.6(1.3-2.0)$ & $1.6(1.2-1.9)$ & $1.5(1.2-1.9)$ & $1.4(1.1-1.8)$ & $<0.001$ & $1.6(1.2-1.9)$ \\
\hline BMI, $\mathrm{kg} / \mathrm{m}^{2}$ & $25.0(22.7-27.8)$ & $25.5(23.1-28.3)$ & $25.5(23.1-28.5)$ & $26.3(23.6-29.5)$ & $<0.001$ & $25.4(23.0-28.3)$ \\
\hline Hypertension, $n(\%)$ & $20,523(46)$ & $27,393(52)$ & $8633(48)$ & $5510(52)$ & $<0.001$ & $61,679(49)$ \\
\hline Type 2 diabetes, $n(\%)$ & $937(2.1)$ & $1949(3.7)$ & $1978(11)$ & $3603(34)$ & $<0.001$ & $7930(6.3)$ \\
\hline $\begin{array}{l}\text { Use of cholesterol-lowering } \\
\text { medication, } n(\%)\end{array}$ & $3748(8.4)$ & $5795(11)$ & $1978(11)$ & $1801(17)$ & $<0.001$ & $12,588(10)$ \\
\hline Current smoker, $n(\%)$ & $25,431(57)$ & $28,973(55)$ & $8633(48)$ & $4980(47)$ & $<0.001$ & $67,973(54)$ \\
\hline $\begin{array}{l}\text { Physical activity, intermediate } \\
\text { or high, } n(\%)\end{array}$ & 30,785 (69) & $32,134(61)$ & $7734(43)$ & $4026(38)$ & $<0.001$ & $74,266(59)$ \\
\hline $\begin{array}{l}\text { Alcohol consumption above } \\
\text { recommended, } n(\%)\end{array}$ & $8923(20)$ & $15,277(29)$ & $8453(47)$ & $5298(50)$ & $<0.001$ & $37,763(30)$ \\
\hline Education $<8$ years, $n(\%)$ & $3525(7.9)$ & $7375(14)$ & $4316(24)$ & $3073(29)$ & $<0.001$ & $17,623(14)$ \\
\hline $\begin{array}{l}\text { Postmenopausal } \\
\quad \text { (women only), } n(\%)\end{array}$ & $15,169(34)$ & $20,544(39)$ & $7554(42)$ & $4026(38)$ & $<0.001$ & $47,833(38)$ \\
\hline
\end{tabular}

Continuous values are median and interquartile range

To convert glucose values to $\mathrm{mg} / \mathrm{dl}$, multiply values in $\mathrm{mmol} / \mathrm{l}$ by 18.0 ; to convert cholesterol values to $\mathrm{mg} / \mathrm{dl}$, multiply values in mmol/l by 38.6 ; and to convert triacylglycerol to $\mathrm{mg} / \mathrm{dl}$, multiply values in $\mathrm{mmol} / \mathrm{l}$ by 88

concentrations and BMI, more often had diabetes $(34 \%$ vs $2.1 \%$ ), used cholesterol-lowering medication, had an alcohol intake above 21/14 units per week and had fewer than 8 years of education, were less physically active and were less often smokers (Table 1). Genotype distributions did not deviate from Hardy-Weinberg expectations overall or in individuals younger or older than age 70 years (all $p>0.05$ ), and none of the variants were in linkage disequilibrium (all $r^{2}<0.4$ ). During up to 43 years of follow-up with a median follow-up of 10.0 years, 2596 individuals developed unspecified dementia (median age at diagnosis 83.0 years [interquartile range 77.1-88.2 years]), 2287 developed Alzheimer's disease (median age 81.3 years [interquartile range 76.1-85.9 years]) and 493 developed vascular dementia (median age 82.8 years [interquartile range $77.0-87.9$ years]).

\section{Characteristics of individuals with a diagnosis of dementia} Compared with individuals with a diagnosis of Alzheimer's disease or vascular dementia, those with a diagnosis of unspecified dementia had higher plasma glucose and LDLcholesterol concentrations, more often had an alcohol intake above recommended, were less often current smokers, were less physically active, less often had hypertension and less often received statin treatment (all $p<0.05$ ). Compared with individuals with vascular dementia, they were also more often women and fewer individuals had had a previous stroke (both $p<0.05$ ). Individuals with unspecified dementia and type 2 diabetes were less often treated with oral diabetes medication, compared with those with Alzheimer's disease or vascular dementia $(p<0.001)$ (ESM Table 1).

Non-fasting glucose and risk of dementia: observational estimates In observational cubic spline analyses, glucose concentrations above the median of $5.2 \mathrm{mmol} / 1$ were associated with linear increases in the risk of unspecified dementia and vascular dementia, but not with the risk of Alzheimer's disease (Fig. 1). In observational multifactorial-adjusted analyses in individuals with a glucose concentration above 7 vs $5-6 \mathrm{mmol} / \mathrm{l}$, the HRs were 1.15 (95\% CI 1.01, 1.32; $p=0.039)$ for unspecified dementia, 0.91 (95\% CI $0.79,1.06 ; p=0.22$ ) for Alzheimer's disease and 1.16 (95\% CI 0.86, $1.55 ; p=0.34)$ for vascular dementia (data not shown). Multifactorialadjusted HRs in individuals with vs without type 2 diabetes were $1.42(95 \%$ CI $1.24,1.63 ; p<0.001)$ for unspecified dementia, 1.11 (95\% CI $0.95,1.29 ; p=0.18)$ for Alzheimer's disease and 1.73 (95\% CI 1.32, 2.27; $p<0.001)$ for vascular dementia (Fig. 1).

Genotypes, non-fasting plasma glucose and risk of dementia: genetic estimates An increasing number of weighted glucose 
Fig. 1 Prospective observational associations of plasma glucose concentration on a continuous scale and in individuals with vs without type 2 diabetes and risk of unspecified dementia (a),

Alzheimer's disease (b) and vascular dementia (c) on a base 2 $\log$ scale, in 125,875 individuals from the general population, the Copenhagen General Population Study and the Copenhagen City Heart Study. Solid lines are multifactorial-adjusted HRs and dashed lines (blue area) indicate 95\% CIs derived from restricted cubic spline Cox regression. $p$ values are from tests for trend across increasing concentrations of glucose above the population median $(5.2 \mathrm{mmol} / \mathrm{l})$. Risk of dementia in individuals with vs without type 2 diabetes is by an age- and sex-adjusted (Model 1) and a multifactorial-adjusted (Model 2) Cox regression model. $* * * p<0.001$ a
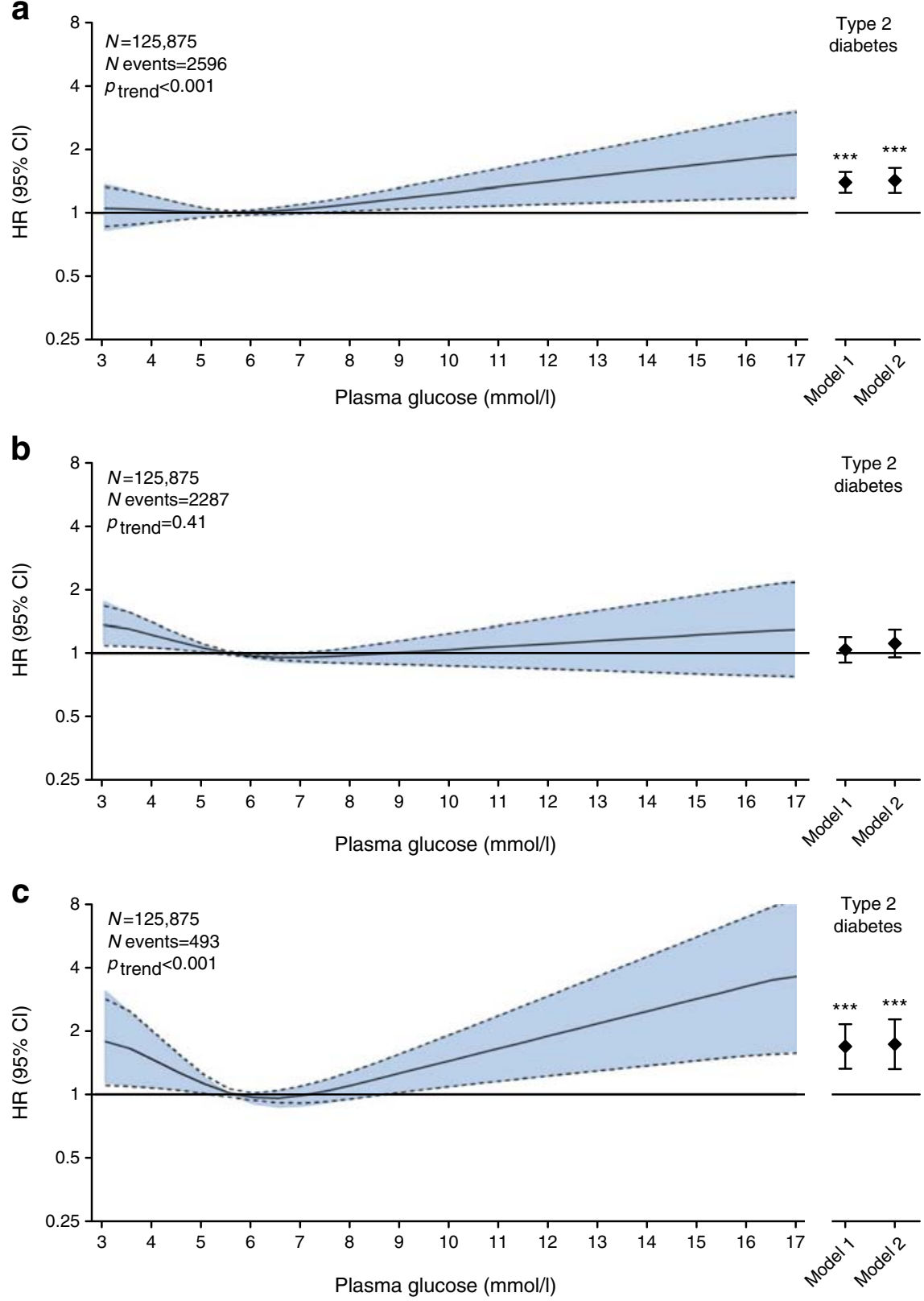

alleles in percentile categories were associated with stepwise higher mean plasma glucose. Individuals in the 95-100th percentile of weighted glucose alleles had $5.1 \%$ higher plasma glucose levels compared with those in the 0-24th percentile. The weighted allele score explained $0.9 \%$ of the variation in non-fasting plasma glucose levels ( $F=80.7$ ) (Fig. 2).

HRs for unspecified dementia, adjusted for age, sex and year of birth, increased with an increasing number of glucoseincreasing alleles ( $p_{\text {trend }}$ across percentile categories $=0.047$ ) with an HR of 1.24 (95\% CI 1.00, 1.53; $p=0.05$ ) in individuals with a weighted glucose allele score in the $95-100$ th percentile vs the $0-24$ th percentile. The weighted allele score was not associated with Alzheimer's disease or vascular dementia (Fig. 2). Similar results were found when the analyses were restricted to individuals not using cholesterol-lowering medication (ESM Fig. 2). Estimates were in the same direction in those without type 2 diabetes mellitus at baseline (ESM Fig. 3), and when using quintiles of the weighted allele score (ESM Fig. 4), although tests for trend across weighted alleles were not significant. Using nonAlzheimer's disease (i.e. all other types of dementia than Alzheimer's disease) as the endpoint gave results in the same direction as those for unspecified dementia; however, the test for trend across ordered categories was not significant (ESM Fig. 5). As dementia is mostly a disease of the old, survival bias may affect risk estimates; however, accounting for the competing risk by death by using a Fine and Gray model gave similar results (ESM Fig. 6). The APOE genotype, which was included as a positive control, showed the known association of the $\varepsilon 43$ and 


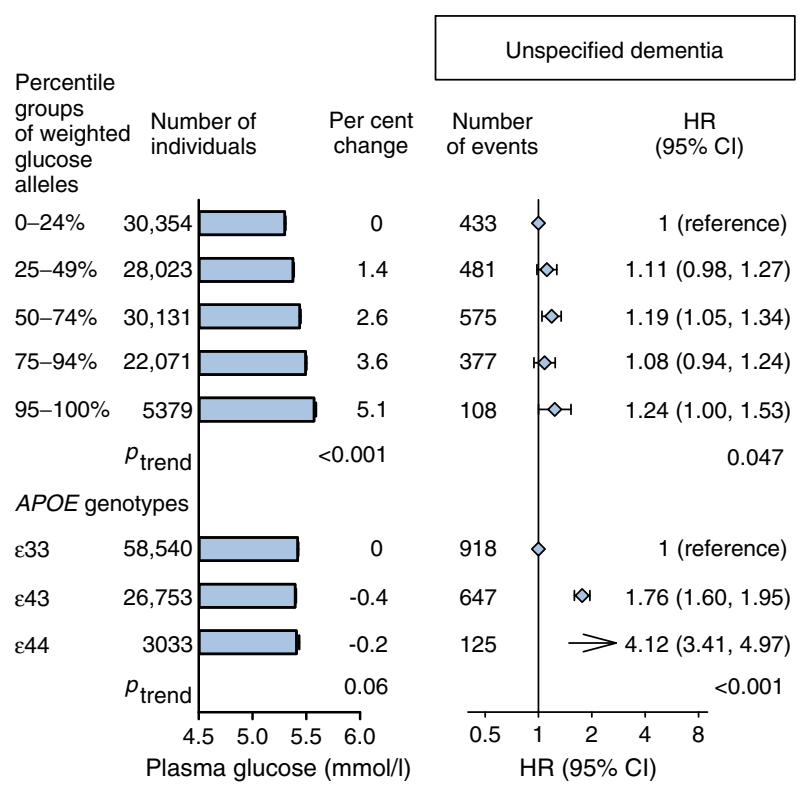

Fig. 2 Prospective risk of unspecified dementia, Alzheimer's disease and vascular dementia shown on a base $2 \log$ scale as a function of percentile groups of a weighted genetic allele score (weighted on the effect on plasma glucose and allele frequency in the Copenhagen studies) adjusted for age, sex and birth year in 115,875 individuals from the general population. Estimates were by Cox regression, excluding individuals with an endpoint before study entry. As a positive control of study power and

$\varepsilon 44$ alleles with the risk of unspecified dementia, Alzheimer's disease and vascular dementia (Fig. 2), confirming the validity of the endpoints used and the study's power to demonstrate positive associations with the endpoints.

Confounding factors Associations between potentially confounding factors and plasma glucose, genetic variants and diagnoses of dementia were tested. Age, sex, hypertension, smoking, physical activity, alcohol consumption, years of education and (for women) menopausal status were all strongly associated with plasma glucose and many were also associated with the risk of unspecified dementia, Alzheimer's disease and vascular dementia (ESM Fig. 7), and may therefore have confounded the observational associations. However, the glucose genotypes were not associated with any major changes in the potential confounders, suggesting that confounding and pleiotropic effects through any of the above factors are unlikely.

Causal effect of glucose on risk of dementia Observationally, a $1 \mathrm{mmol} / \mathrm{l}$ higher non-fasting plasma glucose level had a multifactorial-adjusted HR of 1.04 (95\% CI 1.01, 1.08; $p=$ 0.011 ) for unspecified dementia, 0.97 (95\% CI 0.94, 1.01; $p=$ $0.16)$ for Alzheimer's disease and $1.04(0.97,1.12 ; p=0.24)$ for vascular dementia (Fig. 3).

In genetic, causal analyses, a $1 \mathrm{mmol} / \mathrm{l}$ higher non-fasting plasma glucose level had an RR of $2.40(1.18,4.89 ; p=0.016)$ for unspecified dementia, 1.41 (95\% CI $0.82,2.43 ; p=0.22)$ for Alzheimer's disease and 1.20 (95\% CI 0.82, 1.75; $p=$ 0.36 ) for vascular dementia.

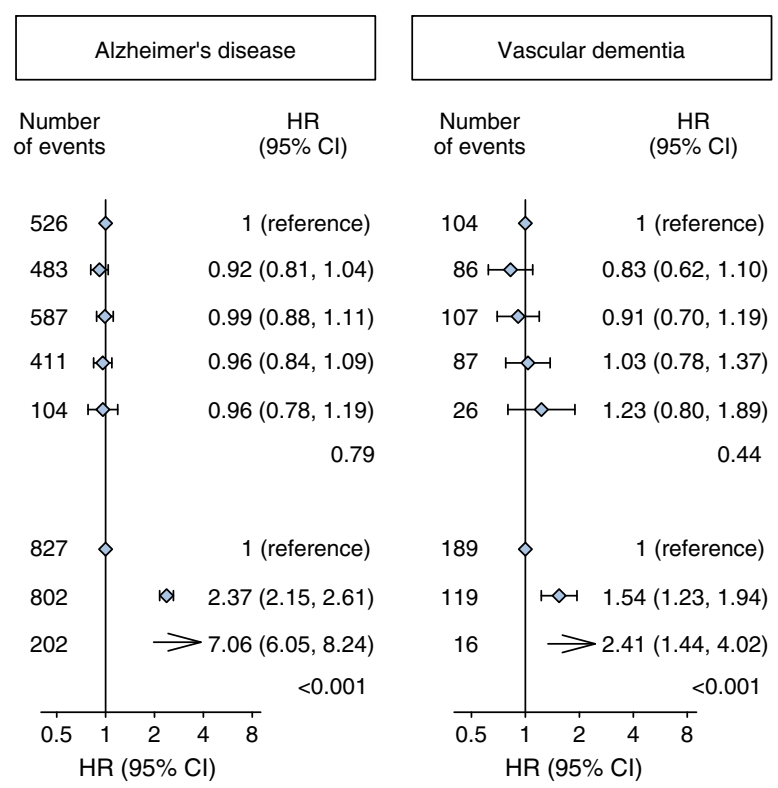

endpoints, risk of dementia is also shown as a function of the APOE $\varepsilon 43$ and $\varepsilon 44$ genotypes compared with the common $\varepsilon 33$ genotype. Arrows denote a point estimate and $95 \%$ CI out of scale. The number of individuals varies slightly due to the availability of genotype data. $p$ values are from tests for trend of HRs across ordered categories. To convert glucose values to $\mathrm{mg} / \mathrm{dl}$, multiply values in $\mathrm{mmol} / \mathrm{l}$ by 18.02

Using summary-level data from MAGIC combined with the ADSP/IGAP/PGC-ALZ/UKB, the RR for Alzheimer's disease was 1.02 (95\% CI 0.92, 1.13; $p=0.42$ ). The RR for Alzheimer's disease using the Copenhagen studies combined with MAGIC + ADSP/IGAP/PGC-ALZ/UKB was 1.03 (95\% CI 0.93, 1.13; $p=$ 0.36) (Fig. 3). In the MAGIC + ADSP/IGAP/PGC-ALZ/UKB studies, the genetic variants showed no indication of directional pleiotropy for variants with low precision (ESM Fig. 8), assumptions for using Egger regression were not violated (ESM Figs 8, 9) and the estimate was not driven by a single genetic variant (ESM Fig. 10).

The power to detect a significant $(\alpha=0.05$ and $\beta=0.80$ ) effect of glucose on the risk of dementia was 1.0 for all endpoints. The risk of ischaemic heart disease, which was included as a positive control for the effect of the genetic instrument, showed the known association of non-fasting plasma glucose levels with the risk of ischaemic heart disease both observationally and genetically (Fig. 3) [9], confirming the validity of the glucose genetic instruments and study power to demonstrate positive associations using these instruments.

\section{Discussion}

In 115,875 individuals from the Copenhagen general population, we found a causal effect of genetically high non-fasting plasma glucose concentrations on the risk of unspecified dementia. Causal genetic effects were not observed for the risk of Alzheimer's disease or of vascular dementia in the 


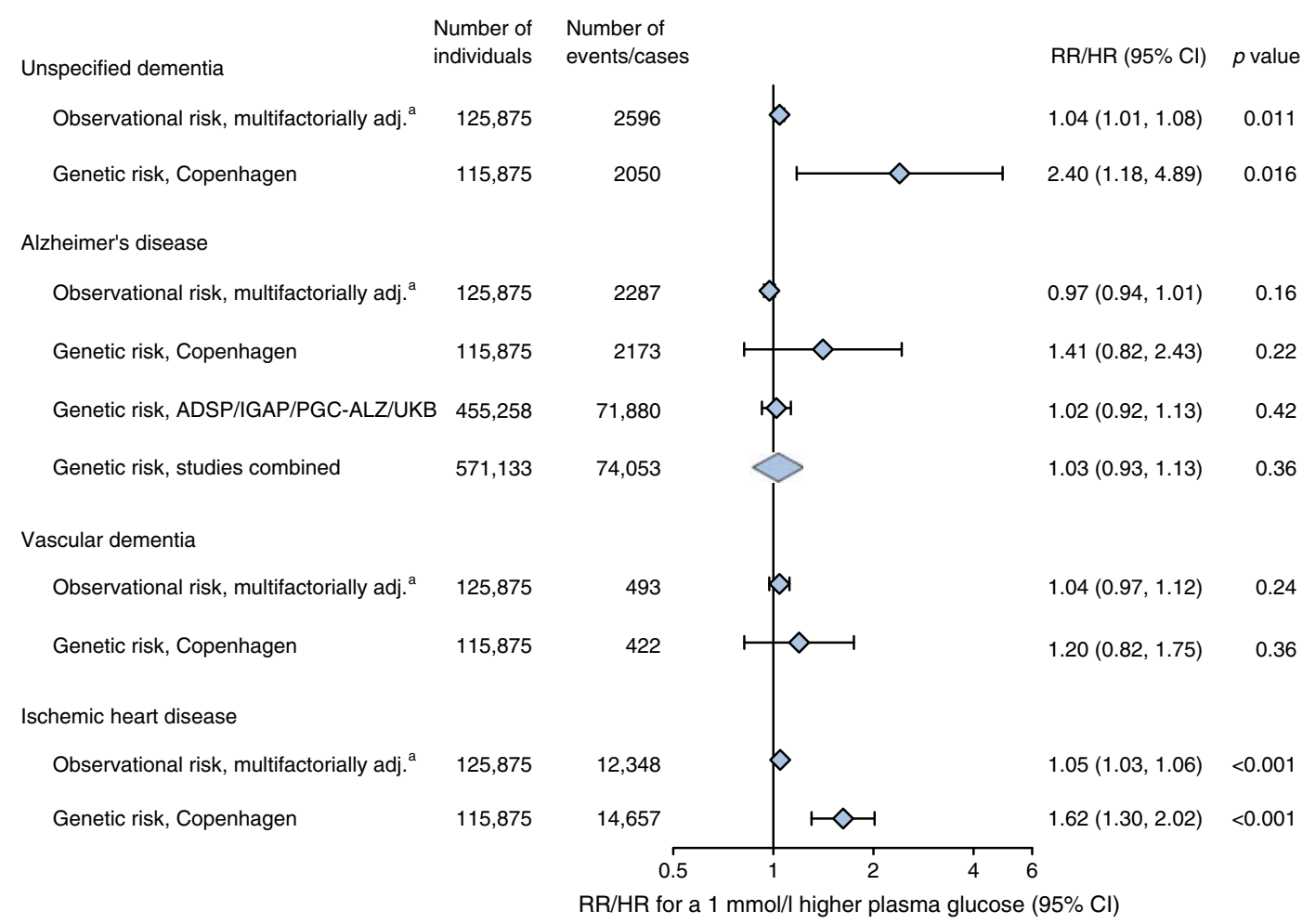

Fig. 3 Risk of unspecified dementia, Alzheimer's disease and vascular dementia for a $1 \mathrm{mmol} / 1$ higher observational and causal, genetically determined plasma glucose concentration. HRs with 95\% CIs for a $1 \mathrm{mmol} / \mathrm{l}$ higher observational plasma glucose concentration were calculated using Cox regression and RRs for genetically higher plasma glucose were derived from instrumental variable analyses. Risk estimates are shown on a base $2 \log$ scale. For Alzheimer's disease, risk was also estimated using summary risk estimates from the PGC-ALZ, IGAP and ADSP, and Alzheimer's disease by proxy (parent with Alzheimer's disease) from the UKB (ADSP/IGAP/PGC-ALZ/UKB) on the same

Copenhagen populations or in the MAGIC + ADSP/IGAP/ PGC-ALZ/UKB participants either alone or combined.

Several studies have examined the observational association between diabetes mellitus and risk of dementia [29-32], and a large meta-analysis [33] found an increased risk of dementia in individuals with vs without diabetes mellitus, with RRs of 1.73 (95\% CI 1.65, 1.82) for all dementia, 1.53 $(95 \%$ CI 1.42, 1.63) for Alzheimer's disease and 2.27 (95\% CI $1.94,2.66)$ for vascular dementia. In addition, one study has examined the observational association between plasma glucose concentrations and risk of dementia, and reported an increased risk of unspecified dementia and Alzheimer's disease combined [1]. In our study, the statistically significant increased risk was observed specifically for unspecified dementia.

A large systematic review has summarised the existing literature on the association between markers of poor glycaemic control (i.e. plasma glucose, $\mathrm{HbA}_{1 \mathrm{c}}$, glucose variability, hypoglycaemic events and fasting insulin) and cognitive function and dementia in patients with diabetes mellitus, and found good evidence for a modest association between genetic variants, but tested in MAGIC using Egger Mendelian randomisation. Estimates for the known observational and causal genetic associations of glucose with the risk of ischaemic heart disease are included as a positive control of the validity of the weighted genetic allele score and study power. $p$ values are for the significance of HRs and RRs. ${ }^{\mathrm{a} B e c}$ ause the observational risks of dementia as a function of glucose concentrations were only linear at glucose concentrations above the population median of $5.2 \mathrm{mmol} / \mathrm{l}$ (Fig. 1), estimates for the observational risk of disease for a $1 \mathrm{mmol} / \mathrm{l}$ higher glucose were limited to glucose concentrations above the median

poor glycaemic control and worse cognitive function, low evidence for associations with the risk of dementia and some evidence that poor glycaemic control is associated with brain abnormalities on MRI [34]. The study also concluded that there are limited data on the effects of glucose-lowering treatment on long-term cerebral outcomes.

The observations in the present study are in concordance with two previous Mendelian randomisation studies of modifiable risk factors for Alzheimer's disease using data from the IGAP, which reported no causal effects of type 2 diabetes, fasting glucose or insulin resistance on the risk of Alzheimer's disease [5, 6]. However, these studies addressed Alzheimer's disease only and did not include data on vascular and unspecified dementia, or observational data.

Elevated plasma glucose concentrations are obviously linked to the presence of diabetes mellitus, and even using the Mendelian randomisation design it is difficult to separate the effect of glucose on the risk of dementia from the multiple effects of diabetes mellitus. In the present study, the genetic variants were associated with lifelong elevated glucose concentrations via known mechanisms, but not with increased 
BMI, hypertension or hyperlipidaemia, confounding factors often associated with diabetes. Excluding individuals with diabetes mellitus at baseline from the causal genetic analyses gave similar results, suggesting that glucose per se has a causal effect on the risk of unspecified dementia, but not on the risk of Alzheimer's disease.

The finding of a causal effect of glucose on the risk of unspecified dementia but not on Alzheimer's dementia is interesting. Evidence from brain autopsy studies has shown that the key features of Alzheimer's disease, such as extracellular deposits of amyloid $\beta$ and intraneuronal aggregates of hyperphosphorylated tau, are not more common in patients with vs without type 2 diabetes [35]. In addition, type 2 diabetes is not associated with cerebrospinal or positron emission tomography biomarkers of increased deposition of cerebral amyloid $\beta$ or tau pathology [36, 37]. This supports the suggestion that high glucose concentrations and diabetes do not contribute directly to the pathology of Alzheimer's disease.

In contrast, there is firm evidence that type 2 diabetes is associated with brain atrophy [38], widespread changes in the microstructure of the white matter and connectivity often related to cognitive dysfunction [38, 39], and an increased burden of cerebrovascular lesions, especially lacunes, but not with an increased burden of large artery infarcts or microinfarcts $[40,41]$. These factors may contribute to cognitive dysfunction and unspecified dementia. This is supported by the present study, which observed that individuals with unspecified dementia, compared with individuals with Alzheimer's disease and vascular dementia, had higher plasma glucose concentrations and, in the presence of type 2 diabetes, were less often treated with oral glucose-lowering drugs ( $15 \%$ vs $33 \%$ and $22 \%$, respectively), suggesting that poor glycaemic control might have contributed to disease risk in these individuals and that improved diabetes diagnosis and management may reduce this risk.

An important limitation of this study is that the endpoints were based on ICD registry codes from hospitals and death certificates from routine clinical practice, and the study therefore only captured individuals in contact with hospitals. This is in contrast to research studies in which all individuals living in one area have been examined and diagnosed using standardised instruments, trained staff and standardised diagnostic methods [42]. Validation of the Danish registry diagnoses has shown that clinical use of the Alzheimer's disease diagnosis is largely limited to individuals fulfilling the diagnostic criteria for this disease, while the diagnoses of vascular dementia and unspecified dementia include individuals with cognitive impairment of mixed causes, including age-related structural brain changes, and those with a previous stroke, atrophy, and large- and small-vessel disease [16-18].

As a sensitivity analysis, we used non-Alzheimer's disease (all other types of dementia than Alzheimer's disease) as an endpoint and obtained results in the same direction as the result for unspecified dementia, although much attenuated and the test for trend across ordered categories was not significant. This suggests that glucose may have a different or no effect in different types of dementia, although our population study was not designed to examine this in further detail. Despite this, data from the ADSP/IGAP/PGC-ALZ/UKB consortium, examining individuals or relatives who have been carefully screened for dementia, showed similar results for Alzheimer's disease as the Danish studies using registry diagnoses. Another potential limitation is the uncertainty of age at onset for dementia diseases. It is commonly accepted that dementia prodromal phases can last for decades. Hence, we cannot exclude that some of the participants receiving a dementia diagnosis during our follow-up time already had dementia pathology at baseline.

As dementia is a disease with onset usually late in life, individuals may die of other causes before developing dementia. This may introduce ascertainment bias, since you cannot participate in a study if you are dead [43]. This is most often an issue in cross-sectional studies. However, in the present prospective study, individuals were followed for up to 43 years with a median of 10 years and diagnoses were retrieved from registers, reducing the risk of low participation because of preexisting conditions. Late onset of disease may also introduce survivor bias; however, genotypes used in the present study were in Hardy-Weinberg equilibrium, suggesting that the genetic instrument was not invalidated by the preferential death of individuals with specific genotypes. Furthermore, using a Fine and Gray model taking the competing risk of death into account did not change the results of the association between weighted glucose alleles and the risk of dementia. If survivor bias was present in our study, it may have reduced the strength of the genetic instrument and resulted in an underestimate of the causal effect in the individual-level analyses of the Mendelian randomisation analysis [44, 45], which could pose a problem for the Alzheimer's disease result. However, the presence of Hardy-Weinberg equilibrium as well as robust supporting results from Fine and Gray models underscore that no strong survivor bias affected our data.

Strengths of this study include our examination of a large, homogenous general population cohort, access to individual participant data of high validity, no losses to follow-up and the use of the Mendelian randomisation design. This approach allowed us to examine potential causal effects of high plasma glucose concentrations, largely without known confounding or reverse causation. The finding of a similar result for the risk of Alzheimer's disease in a two-sample Mendelian randomisation study combining the MAGIC with the ADSP/IGAP/PGC-ALZ/UKB data suggest that the results may apply to other populations, although the fact that $66 \%$ of participants in the ADSP/IGAP/PGC-ALZ/UKB had selfreported disease in a parent and not their own diagnosed disease is a limitation. 
Conclusions and future research In the present study, we used genetic variants that increase plasma glucose concentrations to examine the causal effect of lifelong higher plasma glucose on the risk of unspecified dementia, Alzheimer's disease and vascular dementia in individuals from the general population, and found a causal effect of glucose on the risk of unspecified dementia, but not on that of Alzheimer's disease or vascular dementia. Our finding that high plasma glucose has a causal effect on the risk of unspecified dementia underscores the importance of glycaemic control in patients with diabetes mellitus and potentially also in individuals with impaired glucose tolerance. Future research should address the causal role and potential disease mechanisms of plasma glucose on the risk of unspecified dementia.

Acknowledgements We are indebted to the staff and participants of the Copenhagen General Population Study and the Copenhagen City Heart Study. We also thank the participants of MAGIC and the ADSP/IGAP/ PGC-ALZ/UKB consortium for their generous participation, and the consortia for making their data publicly available. For more information on the consortia data, please refer to the ESM Methods/ Acknowledgements.

Data availability Due to participant confidentiality, only data presented in the paper and the ESM are available.

Funding This study was supported by The Danish Council for Independent Research, Medical Sciences; and Herlev and Gentofte Hospital, Copenhagen University Hospital.

Authors' relationships and activities The authors declare that there are no relationships or activities that might bias, or be perceived to bias, their work.

Contribution statement All authors designed the study, acquired the data, performed the analyses, interpreted the findings, wrote and revised the report, and contributed to data collection, data preparation or both. MB drafted the manuscript and all authors contributed to critical reading and revision of the draft report, and all authors approved the final version to be published. MB is the guarantor of this work and, as such, had full access to all the data in the study and takes responsibility for the integrity of the data and the accuracy of the data analysis.

\section{References}

1. Crane PK, Walker R, Hubbard RA et al (2013) Glucose levels and risk of dementia. N Engl J Med 369(6):540-548. https://doi.org/10. 1056/NEJMoa1215740

2. Lawlor DA, Harbord RM, Sterne JA, Timpson N, Davey SG (2008) Mendelian randomization: using genes as instruments for making causal inferences in epidemiology. Stat Med 27(8):1133-1163. https://doi.org/10.1002/sim.3034

3. Smith GD, Ebrahim S (2003) 'Mendelian randomization': can genetic epidemiology contribute to understanding environmental determinants of disease? Int J Epidemiol 32:1-22

4. Benn M, Nordestgaard BG (2018) From genome-wide association studies to Mendelian randomization: novel opportunities for understanding cardiovascular disease causality, pathogenesis, prevention, and treatment. Cardiovasc Res 114(9):1192-1208. https://doi.org/ $10.1093 / \mathrm{cvr} / \mathrm{cvy} 045$

5. Larsson SC, Traylor M, Malik R et al (2017) Modifiable pathways in Alzheimer's disease: Mendelian randomisation analysis. BMJ 359:j5375

6. Ostergaard SD, Mukherjee S, Sharp SJ et al (2015) Associations between potentially modifiable risk factors and Alzheimer disease: a Mendelian randomization study. PLoS Med 12:e1001841

7. Kuzma E, Hannon E, Zhou A et al (2018) Which risk factors causally influence dementia? A systematic review of Mendelian randomization studies. J Alzheimers Dis 64(1):181-193. https:// doi.org/10.3233/JAD-180013

8. Benn M, Nordestgaard BG, Frikke-Schmidt R, Tybjaerg-Hansen A (2017) Low LDL cholesterol, PCSK9 and HMGCR genetic variation, and risk of Alzheimer's disease and Parkinson's disease: Mendelian randomisation study. BMJ 357:j1648

9. Benn M, Tybjaerg-Hansen A, McCarthy MI, Jensen GB, Grande P, Nordestgaard BG (2012) Nonfasting glucose, ischemic heart disease, and myocardial infarction: a Mendelian randomization study. J Am Coll Cardiol 59(25):2356-2365. https://doi.org/10. 1016/j.jacc.2012.02.043

10. McCarthy MI, Abecasis GR, Cardon LR et al (2008) Genome-wide association studies for complex traits: consensus, uncertainty and challenges. Nat Rev Genet 9(5):356-369. https://doi.org/10.1038/ $\operatorname{nrg} 2344$

11. Morris AP, Voight BF, Teslovich TM et al (2012) Large-scale association analysis provides insights into the genetic architecture and pathophysiology of type 2 diabetes. Nat Genet 44:981-990

12. Scott RA, Lagou V, Welch RP et al (2012) Large-scale association analyses identify new loci influencing glycemic traits and provide insight into the underlying biological pathways. Nat Genet 44(9): 991-1005. https://doi.org/10.1038/ng.2385

13. Dupuis J, Langenberg C, Prokopenko I et al (2010) New genetic loci implicated in fasting glucose homeostasis and their impact on type 2 diabetes risk. Nat Genet 42(2):105-116. https://doi.org/10. 1038/ng.520

14. Jansen IE, Savage JE, Watanabe K et al (2019) Genome-wide metaanalysis identifies new loci and functional pathways influencing Alzheimer's disease risk. Nat Genet 51(3):404-413. https://doi. org/10.1038/s41588-018-0311-9

15. Frikke-Schmidt R, Nordestgaard BG, Thudium D, Moes Gronholdt ML, Tybjaerg-Hansen A (2001) APOE genotype predicts AD and other dementia but not ischemic cerebrovascular disease. Neurology 56(2):194-200. https://doi.org/10.1212/wnl.56.2.194

16. Rasmussen KL, Tybjaerg-Hansen A, Nordestgaard BG, FrikkeSchmidt R (2015) Plasma levels of apolipoprotein E and risk of dementia in the general population. Ann Neurol 77(2):301-311. https://doi.org/10.1002/ana.24326

17. Phung TK, Andersen BB, Hogh P, Kessing LV, Mortensen PB, Waldemar G (2007) Validity of dementia diagnoses in the Danish hospital registers. Dement Geriatr Cogn Disord 24:220-228

18. Rasmussen KL, Tybjaerg-Hansen A, Nordestgaard BG, FrikkeSchmidt R (2018) Plasma apolipoprotein E levels and risk of dementia: a Mendelian randomization study of 106,562 individuals. Alzheimers Dement 14(1):71-80. https://doi.org/10.1016/j.jalz. 2017.05.006

19. World Health Organization, International Society of Hypertension Writing Group (2003) 2003 World Health Organization (WHO)/ International Society of Hypertension (ISH) statement on management of hypertension. J Hypertens 21(11):1983-1992. https://doi. org/10.1097/00004872-200311000-00002

20. World Health Organization (2006) Definition and diagnosis of diabetes mellitus and intermediate hyperglycemia. Report of a WHO/IDF consultation. WHO, Geneva

21. StataCorp LP (2016) Stata Statistical Software Release 14.2. StataCorp LP, College Station, TX 
22. Akaike H (1998) Information theory and an extension of the maximum likelihood principle. In: Parzen E, Tanabe K, Kitagawa G (eds) Selected papers of Hirotugu Akaike. Springer, New York, pp 199-213

23. Baum C, Schaffer M, Stillman S (2003) Instrumental variables and GMM: estimation and testing. Stata J 3:1-31

24. Nichols A (2007) IVPOIS: Stata module to estimate an instrumental variables Poisson regression via GMM. Available at: http://ideas. repec.org/c/boc/bocode/s456890.html. Accessed 14 Feb 2020

25. Burgess S, Butterworth A, Thompson SG (2013) Mendelian randomization analysis with multiple genetic variants using summarized data. Genet Epidemiol 37(7):658-665. https://doi. org/10.1002/gepi.21758

26. Burgess S, Bowden J, Fall T, Ingelsson E, Thompson SG (2017) Sensitivity analyses for robust causal inference from Mendelian randomization analyses with multiple genetic variants. Epidemiology 28(1):30-42. https://doi.org/10.1097/EDE. 0000000000000559

27. Bowden J, Davey SG, Burgess S (2015) Mendelian randomization with invalid instruments: effect estimation and bias detection through Egger regression. Int J Epidemiol 44(2):512-525. https:// doi.org/10.1093/ije/dyv080

28. Brion MJ, Shakhbazov K, Visscher PM (2013) Calculating statistical power in Mendelian randomization studies. Int J Epidemiol 42(5):1497-1501. https://doi.org/10.1093/ije/dyt179

29. Kloppenborg RP, van den Berg E, Kappelle LJ, Biessels GJ (2008) Diabetes and other vascular risk factors for dementia: which factor matters most? A systematic review. Eur J Pharmacol 585(1):97108. https://doi.org/10.1016/j.ejphar.2008.02.049

30. Meng XF, Yu JT, Wang HF et al (2014) Midlife vascular risk factors and the risk of Alzheimer's disease: a systematic review and metaanalysis. J Alzheimers Dis 42(4):1295-1310. https://doi.org/10. 3233/JAD-140954

31. Xu W, Tan L, Wang HF et al (2015) Meta-analysis of modifiable risk factors for Alzheimer's disease. J Neurol Neurosurg Psychiatry 86:1299-1306

32. Biessels GJ, Staekenborg S, Brunner E, Brayne C, Scheltens P (2006) Risk of dementia in diabetes mellitus: a systematic review. Lancet Neurol 5(1):64-74. https://doi.org/10.1016/S14744422(05)70284-2

33. Chatterjee S, Peters SA, Woodward M et al (2016) Type 2 diabetes as a risk factor for dementia in women compared with men: a pooled analysis of 2.3 million people comprising more than 100,000 cases of dementia. Diabetes Care 39:300-307

34. Geijselaers SLC, Sep SJS, Stehouwer CDA, Biessels GJ (2015) Glucose regulation, cognition, and brain MRI in type 2 diabetes: a systematic review. Lancet Diabetes Endocrinol 3(1):75-89. https:// doi.org/10.1016/S2213-8587(14)70148-2

35. Arvanitakis Z, Schneider JA, Wilson RS et al (2006) Diabetes is related to cerebral infarction but not to AD pathology in older persons. Neurology 67:1960-1965

36. Gottesman RF, Albert MS, Alonso A et al (2017) Associations between midlife vascular risk factors and 25-year incident dementia in the Atherosclerosis Risk in Communities (ARIC) cohort. JAMA Neurol 74(10):1246-1254. https://doi.org/10.1001/jamaneurol. 2017.1658

37. Moran C, Beare R, Phan TG et al (2015) Type 2 diabetes mellitus and biomarkers of neurodegeneration. Neurology 85(13):11231130. https://doi.org/10.1212/WNL.0000000000001982

38. Biessels GJ, Reijmer YD (2014) Brain changes underlying cognitive dysfunction in diabetes: what can we learn from MRI? Diabetes 63(7):2244-2252. https://doi.org/10.2337/db14-0348

39. Moran C, Beare R, Phan T et al (2017) Neuroimaging and its relevance to understanding pathways linking diabetes and cognitive dysfunction. J Alzheimers Dis 59(2):405-419. https://doi.org/10. 3233/JAD-161166

40. Abner EL, Nelson PT, Kryscio RJ et al (2016) Diabetes is associated with cerebrovascular but not Alzheimer's disease neuropathology. Alzheimers Dement 12(8):882-889. https://doi.org/10.1016/j. jalz.2015.12.006

41. Pruzin JJ, Schneider JA, Capuano AW et al (2017) Diabetes, hemoglobin $\mathrm{A} 1 \mathrm{C}$, and regional Alzheimer disease and infarct pathology. Alzheimer Dis Assoc Disord 31(1):41-47. https://doi.org/10.1097/ WAD.0000000000000172

42. Ikram MA, Brusselle GGO, Murad SD et al (2017) The Rotterdam Study: 2018 update on objectives, design and main results. Eur J Epidemiol 32(9):807-850. https://doi.org/10.1007/s10654-0170321-4

43. Hernan MA, Alonso A, Logroscino G (2008) Cigarette smoking and dementia: potential selection bias in the elderly. Epidemiology 19(3):448-450. https://doi.org/10.1097/EDE.0b013e31816bbe14

44. Smit RAJ, Trompet S, Dekkers OM, Jukema JW, le Cessie S (2019) Survival bias in Mendelian randomization studies: a threat to causal inference. Epidemiology 30:813-816

45. Vansteelandt S, Dukes O, Martinussen T (2018) Survivor bias in Mendelian randomization analysis. Biostatistics 19(4):426-443. https://doi.org/10.1093/biostatistics/kxx050

Publisher's note Springer Nature remains neutral with regard to jurisdictional claims in published maps and institutional affiliations. 